\title{
Beratung in der pränatalen Diagnostik: Eine Nationalfondsstudie über Entscheid- unterstützungssysteme
}

\author{
D. C. Hürlimann ${ }^{a}$, R. Baumann-Hölzle
}

Schwangerschaften werden heutzutage medizinisch intensiv betreut. Zum Angebot in der Pränatalen Diagnostik gehören technische Hilfsmittel wie Ultraschall und ein vielfältiges Testangebot. Die Entscheidung betreffend der Inanspruchnahme eines Tests stellt eine schwangere Frau und ihren Partner vor eine komplexe Situation.

Mit dem hier beschriebenen Projekt soll der Beratung im pränatalen Screening und in der Diagnostik ${ }^{1}$ mehr Gewicht verliehen werden. Im Rahmen eines Nationalfondsprojektes soll die auf das spezifische Problemfeld der vorgeburtlichen Untersuchung ausgerichtete Beratung untersucht und analysiert werden. Ein von einem interdisziplinären Fachgremium erstellter Leitfaden im Sinne eines Beratungsinstrumentes wird evaluiert.

Das evaluierte und allenfalls angepasste Beratungsinstrument wird anschliessend in der ärztlichen Praxis in einem möglichst breiten Rahmen eingesetzt.

Zur Teilnahme an der geplanten Evaluation des Leitfadens werden praktizierende Ärztinnen und Ärzte, welche Schwangerschaftsbegleitungen machen, gesucht. Die Evaluation umfasst zwei Analysen des Beratungsprozesses, eine vor der Einführung des Leitfadens und eine danach. Die Einführung geschieht mittels einer Schulung der teilnehmenden Ärztinnen und Ärzte, durchgeführt durch die Frauenspitäler Basel und Zürich (SGGG-Fortbildungspunkte sind beantragt).

\section{a Interessenverbindungen: National- fondsprogramm NFP 51, Gruppe Genetik unter der Leitung von Prof. Dr. med. Hansjakob Müller.}

1 Begriffsabgrenzung: Während das pränatale Screening angewandt wird, um eine Risikosituation in der Schwangerschaft zu erkennen, dient die pränatale Diagnostik dazu, eine offensichtliche Risikokonstellation wie das Alter der Schwangeren oder ein früheres Kind mit Down-Syndrom abzuklären.

Korrespondenz:

Denise C. Hürlimann

St. Wolfgangstrasse 29

CH-6331 Hünenberg

Tel. 0793219992

E-Mail: dch@bluemail.ch

Dr. theol. Ruth Baumann-Hölzle Institut Dialog Ethik

Interdisziplinäres Institut für Ethik im Gesundheitswesen

Sonneggstrasse 88

CH-8006 Zürich

Tel. 012524201

Fax 012524213

E-Mail: info@dialog-ethik.ch

\section{Problemstellung und Zielsetzung}

Mit dem zunehmenden wissenschaftlichen Fortschritt in der Medizin, speziell in der Genetik, gelangt eine Ärztin / ein Arzt im Bereich des pränatalen Screenings und der Diagnostik an eine nicht unproblematische Doppelfunktion, wenn sie/er sowohl Entscheidangebot wie auch Entscheidvollzug vereinen. Die kürzlich aktuell gewordenen Haftpflichtfälle in verschiedenen europäischen Staaten dokumentieren leider die Unsicherheit auch auf ärztlicher Seite. Ein Beratungsinstrument soll einer Ärztin oder einem Arzt in diesem Zusammenhang Sicherheit vermitteln. Für die schwangere Frau oder das Paar liegt die Schwierigkeit einerseits darin, dass der Entscheid nicht nur das eigene Leben betrifft und soziale Folgen hat, sondern auch das Bewusstsein in einer Gesellschaft verändern kann und von ihm geprägt ist. Anderseits erfolgt ein solcher Entscheid meist unter Zeitdruck, Entscheidunsicherheit und einer Entscheidambivalenz. Die Absicht der nicht-direktiven Beratung ist es, dass die Patientin oder das Paar zu einem informierten Entscheid gelangen kann, mit dem sie/es noch Jahre leben kann. Dem Risikoaspekt wird sowohl von seiten der Patientin oder des Paares wie auch von ärztlicher Seite Rechnung getragen. Das Schwergewicht der Untersuchung liegt bei den Ärztinnen und Ärzten, wenngleich Patientinnen auch involviert sein werden.

Obwohl im Bereich der medizinischen Literatur einiges zum Thema Pränatale Diagnostik publiziert wurde, ist der Stand der Literatur zum Thema Beratung und psychologischer Entscheidprozess noch dürftig. Es bestehen einige kurze Studien, in welchen die psychologischen Implikationen bei der Untersuchung spezifischer Genmutationen (z. B. Chorea Huntington) mitbehandelt werden. In der Schweiz wurde 1997 eine Studie zu den quantitativen Angaben über pränatale Diagnostik gemacht, die psychologischen Auswirkungen sind in der Schweiz allerdings noch weitgehend unerforscht. Da die Publikationen des englischen Kulturraums nicht bedingungslos auf Deutschschweizer Patienten angewendet werden können, ist die Realisierung unseres Projektes nötig und sinnvoll. 2001 wurde auf Auftrag des Zentrums für Technologiefolgenabschätzung und des Bundesamtes für Sozialversicherungen eine Studie zu den psychosozialen Aspekten in der Ultraschalluntersuchung publiziert. Diese Studie liefert Empfehlungen und kann als Ausgangsbasis für unsere Studie, welche verschiedene pränatale Testmethoden einbezieht, betrachtet werden.

\section{Zur Situation der Beratung in der pränatalen Diagnostik}

Ein erster Kontakt mit dem Untersuchungsumfeld in Form von explorativen Interviews mit Fachexperten und -expertinnen hat die nachfolgenden Rahmenbedingungen für die Beratung der pränatalen Diagnostik im ärztlichen Setting aufgezeigt. Bei der Auswahl der Interviewpartner wurde darauf geachtet, dass möglichst viele Expertengruppen zur Sprache kamen (Ärztinnen, Ärzte, Patientinnen, praktizierende Gynäkologen, Spitalärzte, Berater von Schwangerschaftsberatungen, Ärzte verschiedener Regionen und hierarchischer Ebenen). 
- Zwischen den Beratungen in einer Universitätsklinik (Poliklinik) und der Beratung in einer gynäkologischen Praxis bestehen erwartungsgemäss Unterschiede im Patientengut einerseits, aber auch im Vertrauensverhältnis zwischen Ärztin/Arzt und Patientin anderseits. Ein praktischer Arzt oder eine Ärztin kennt eine Patientin häufig bereits jahrelang, bevor diese schwanger wird.

- Beratungen finden in einem eng begrenzten zeitlichen Rahmen und überdies über verschiedene Wochen verteilt und damit fragmentiert statt.

- Da das Angebot an Methoden der pränatalen Diagnostik, welche standardmässig von einem Arzt oder einer Ärztin angeboten werden, unterschiedlich ist (z. B. ist der AFP-PlusTest standardmässig oder nur auf ausdrücklichen Wunsch im Angebot), sind auch die Beratungsbedürfnisse vom Inhalt her unterschiedlich.

- Eine feinfühlige Kommunikation wird von verschiedenen Seiten her immer wieder als sehr wichtig erachtet. Wichtig scheint diese insbesondere bei der Interpretation von Ultraschallbildern, der Besprechung von Testergebnissen und bei Fragen seitens der Frauen. Neben dem Sprachgebrauch verlangt auch der Umgang mit dem Testergebnis von einem Berater viel Fingerspitzengefühl und Zeit.

- Der Umgang mit einem allfällig positiven Resultat muss bereits vor dem Test bedacht werden. Ist ein erster Test positiv, kann sich eine Frau während der ganzen Schwangerschaft Sorgen machen, auch wenn die nachfolgenden Tests negativ sind. Ein positives Ergebnis lässt gewisse Frauen kaum mehr los. Dies ist deshalb bedenkenswert, da ein Test falsch-positive Resultate aufzeigen kann (z. B. im Triple-Test sind von 100 positiven Resultaten für Trisomie 21 deren 98 falsch-positiv).

- Anderseits bedeutet ein positives Testergebnis, wenn es auch in verschiedenen Untersuchungen bestätigt wurde, noch nicht automatisch, dass die Schwangerschaft abgebrochen wird.

- Schulungen und Kompetenzerwerb in Beratungen finden bei Ärztinnen und Ärzten kaum statt, sind hingegen von zahlreichen Ärztinnen und Ärzten erwünscht.

- Eine optimale Beratung muss aufgrund der erwähnten Sachzwänge zeitsparend durchgeführt werden können.

- Das subjektive Empfinden in der Schwangerschaft ist stark geprägt von körperlichen Beschwerden wie Unwohlsein, Müdigkeit, Energielosigkeit, hormonellen Umstellungen und der Übernahme der Verantwortung für das Kind (Rauchen, Alkohol usw.). Auf der andern Seite ist die Freude über das Kind sehr gross. Für die pränatale Diagnostik und die Herausforderung der Entscheidung darüber scheint bei einigen schwangeren Frauen beinahe kein Raum übrigzubleiben.

- Zum Ultraschallbild: Gelegentlich wird darauf ausgewichen, da es für einen Arzt oder eine Ärztin dankbarer sein kann als Beratung, nicht nur aus finanziellen Gründen. Ein Bild stellt etwas dar, eine Patientin sieht ihr Kind darauf. Ausserdem ist es beruhigend, alle Beine und Arme zu sehen. Anderseits kann ein Ultraschallbild traumatisierend wirken, da ein Kind «unfertig» aussieht und ein Ultraschallbild für Laien schwierig zu interpretieren ist.

- Die Beratung zur pränatalen Diagnostik wird mehrheitlich Frauen gegenüber erteilt, selten ist ihr Lebenspartner anwesend, wenn kein spezifisches Verdachtsmoment vorliegt. Da viele Paare sich vor einer Schwangerschaft nicht stark mit dem Thema pränatale Diagnostik und Behinderung beschäftigen, könnte man folgern, dass der Entscheid, pränatale Diagnostik in Anspruch zu nehmen oder nicht, grösstenteils von der Frau gefällt wird, manchmal nach einer Diskussion mit ihrem Partner zu Hause zwischen zwei Konsultationen bei der Ärztin oder beim Arzt.

- Der Informationsgehalt von Broschüren und Dokumentationen ist verbesserungsfähig. Ein einheitlicher Einsatz von Dokumentationen ist deshalb sinnvoll, da schwangere Frauen untereinander einen Austausch pflegen.

- Ein Stufenkonzept der Informationsvermittlung scheint sinnvoll. Damit kann dem unterschiedlichen Wissensstand und -bedürfnis Rechnung getragen werden.

\section{Der Leitfaden und seine Einführung}

Um Handeln unter Unsicherheit zu unterstützen, braucht man Methoden der Entscheidfindung, und das können Unterstützungssysteme wie Heuristiken sein. Ein von einem interdisziplinären Fachgremium zusammengestellter Beratungsleitfaden der pränatalen Diagnostik für Ärztinnen und Ärzte zur Begleitung der schwangeren Frau und ihres Partners wird in der ärztlichen Praxis implementiert und begleitet, sofern die Analyse des Beratungsprozesses die Notwendigkeit hierfür aufzeigt. Dieses Beratungsinstru- 
ment wurde mit verschiedensten Berufsgruppen unter der Leitung des Vereins Ganzheitliche Beratung und kritische Information zu pränataler Diagnostik, Dr. theol. Ruth Baumann-Hölzle, Dr. med. Suzanne Braga und Prof. Dr. med. Roland Zimmermann erarbeitet und an zwei Tagungen besprochen. Die Einführung wird von einer Schulung durch die beiden medizinischen Zentren in Basel und Zürich (Dr. med. Sevgi Tercanli und Dr. med. Judit Pôk) begleitet.

Die Evaluation des Leitfadens erfolgt innerhalb einer Dissertation am Psychologischen Institut der Universität Zürich. Das Projekt ist ausserdem Teil eines interdisziplinären Nationalfondsprojekts, das im Nationalfondsprogramm 51 «Integration und Ausschluss» im Bereich Genetik stattfindet. Es werden regelmässig Kolloquien für den Austausch der Forschungsresultate durchgeführt. Die Leitung der Gruppe obliegt Prof. Hansjakob Müller, Leiter der Abteilung Medizinische Genetik, UKBB, Departement der Klinisch-Biologischen Wissenschaften, Universität Basel.

Der zu evaluierende Leitfaden ist vorwiegend medizinisch geprägt und beinhaltet die Implikationen aus der gängigen Rechtssprechung in der Schweiz. Inwieweit psychologische Aspekte zusätzlich integriert werden müssen, wird die geplante Evaluation und die Analyse des Beratungsprozesses zeigen. Ziel des Projektes ist nicht die Frage nach dem medizinisch Machbaren oder dem ethisch-moralisch Sinnvollen, sondern vielmehr nach den psychologischen Prozessen der Beratung und Entscheidung.

Daneben erfolgt die Umsetzung und praktische Anwendung des Leitfadens innerhalb eines Paketes von drei Massnahmen, nämlich mit

Abbildung 1

Untersuchungsdesign zur Evaluation des Beratungsleitfadens.

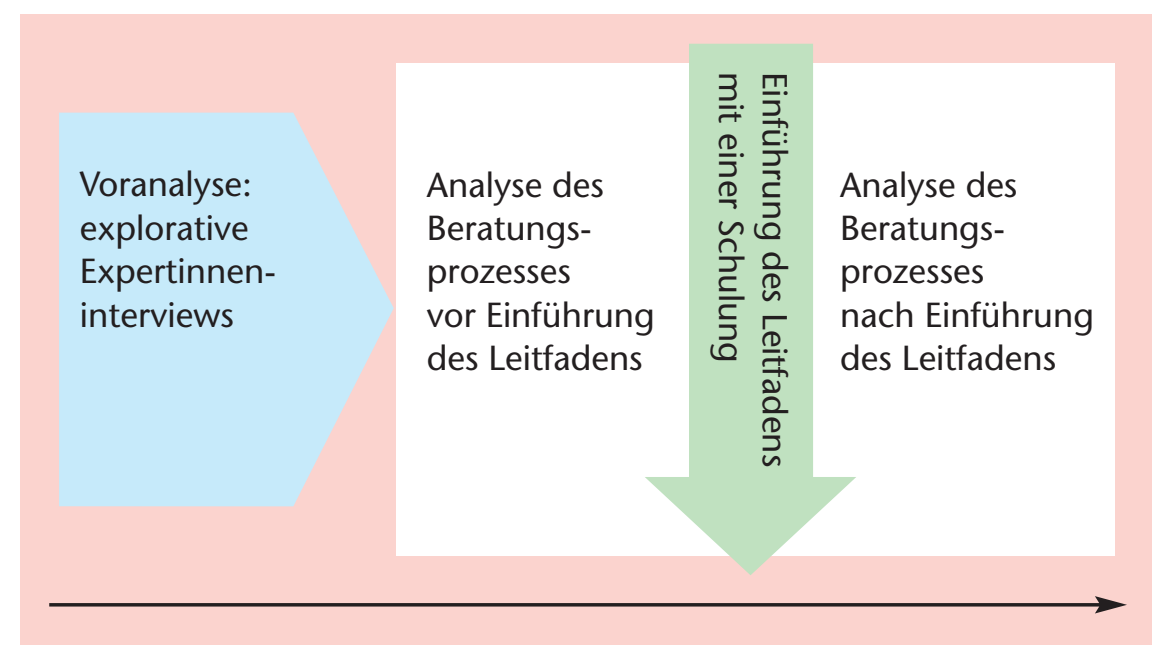

einem zum Beratungsinstrument abgestimmten Film und einer Broschüre für das zu beratende Paar (Herausgeber: Verein Ganzheitliche Beratung und kritische Information zu pränataler Diagnostik). Mit den Verantwortlichen dieses DreierPakets, Dr. med. Suzanne Braga, Dr. theol. Ruth Baumann-Hölzle und Prof. Dr. med. Roland Zimmermann, wird intensiv zusammengearbeitet.

\section{Evaluation des Leitfadens}

Die verbreitete Logik in der Einführung eines Modells oder Leitfadens, die dem Schema «Einführung - Evaluation - Praxis» folgt, wird in diesem Projekt erweitert, indem man den Evaluationsanspruch nicht als abschliessendes summatives, sondern vielmehr als formatives Geschehen betrachtet. Da ein solches Beratungsinstrument nur Sinn macht, wenn ein Bedürfnis der Anwender und Nutzniesser besteht, wird der Beratungsprozess in einer Vorevaluation zuerst dahingehend untersucht. Das Projekt beinhaltet neben der Analyse des Beratungsprozesses eine Beurteilung der Konzeption, Umsetzung, Ausgestaltung und des Nutzens des Leitfadens sowie der begleitenden Schulung. Man muss davon ausgehen, dass der Stand des Wissens zu einer Veränderung des pränatalen Screenings und der Diagnostik führt. Deshalb muss ein solcher Leitfaden und die Schulung von Fachpersonen die Entwicklung im pränatalen Screening und in der Diagnostik laufend integrieren und widerspiegeln können.

\section{Das Untersuchungsdesign}

Während einer Erhebung der Ist-Situation (vor der Einführung des Beratungsinstrumentes in Form des Leitfadens) wird der Beratungsprozess analysiert und es wird der Frage nachgegangen, wie die Akzeptanz eines solchen Leitfadens per se bei den Ärzten und Ärztinnen ist. Aus diesen Punkten ergibt sich dann die Notwendigkeit für die Einführung eines Leitfadens, indem anhand zuvor definierter Kriterien der Beratungsprozess analysiert wird. Nach der allfälligen Einführung des Leitfadens fokussiert das Projekt auf die Frage, wie hilfreich der Leitfaden bei der Entscheidfindung empfunden wird, und beschäftigt sich mit den psychologischen Auswirkungen der begleitenden Schulung und des Leitfadens. Die Veränderung des Arzt-Patientinnen-Verhältnisses ist im Rahmen des Entscheidfindungsprozesses von besonderem Interesse. Die Einführung des Beratungsinstrumentes erfolgt mittels Schu- 
lung bei den Ärztinnen und Ärzten. Neben der Schulung von Beratungskompetenz wird der Leitfaden so praxisnah eingeführt.

Die Untersuchung wird bei niedergelassenen Ärztinnen und Ärzten in Gynäkologie und Allgemeinmedizin ${ }^{2}$ sowie deren Patientinnen durchgeführt. Der Leitfaden wird zuerst in der Region Zürich eingeführt. Ein halbes Jahr später erfolgt die Einführung in der Region Basel. Dazu können die Erfahrungen von der Einführung in Zürich ausgewertet und der Leitfaden sowie dessen Einführung optimiert werden. Die Poliklinik des Departements Frauenheilkunde im Universitätsspital Zürich, USZ (Prof. Dr. med. R. Zimmermann, Dr. med. J. Pôk), rekrutiert in enger Zusammenarbeit mit Denise Hürlimann die Ärztinnen und Ärzte in der Region Zürich und übernimmt deren Schulung.

Das Ziel der Evaluation ist es einerseits, nach der Untersuchung über die Notwendigkeit eines Instrumentes die Beratung zuerst ohne und dann mittels Leitfaden zu beschreiben, und anderseits, Ergebnisse und Entwicklungen laufend in die Schulung und den Leitfaden integrieren zu können (formativer Aspekt).

Als testmethodisch besonders wertvoll kann die Möglichkeit der Vorevaluation vor Einführung der Schulung und des Leitfadens angesehen werden. Die Implikationen eines entscheidunterstützenden Beratungsinstrumentes und der Schulung von Ärztinnen und Ärzten können dementsprechend eruiert werden.

\section{Methoden der Analyse des Beratungsprozesses}

Das Ziel einer Beratung ist, dass eine schwangere Frau und ihr Partner einen informierten Entscheid bezüglich der Inanspruchnahme von möglichen Tests und Untersuchungen in der pränatalen Diagnostik fassen können. Diese Beratung soll zuerst vor der Einführung des Frameworks zur Entscheidunterstützung (d.h. des Leitfadens) und anschliessend nochmals analysiert werden. Damit ist ein Vorher-Nachher-Vergleich möglich.

Der Gegenstand der Analyse der Beratung ist es, die Auswirkungen, welche das Framework auf die Beratung hat, erfassen zu können. Wir gehen von folgenden Auswirkungen aus:
- Standardisierung des Beratungsprozesses;

- Unsicherheit auf seiten der Ärztinnen/Ärzte und Patientinnen reduzieren;

- Straffung des Beratungsprozesses;

- Beratung im Sinne der nicht-direktiven Beratung ermöglichen;

- Unterstützung des psychologischen Entscheidprozesses auf seiten der Patientinnen.

Mit der Analyse der Beratung sollen nicht sämtliche Aspekte einer Arzt-Patienten-Beziehung, sondern vielmehr die Interventionsmethodik des Frameworks abgedeckt werden.

Die Analyse muss dabei Aussagen machen über:

- die Dynamik der Beratung;

- den Prozess der Beratung;

- die Vermittlung von Wissenselementen seitens der Ärztin / des Arztes;

- den Umgang mit psychologischen Konstrukten auf seiten der Ärztinnen/Ärzte und Patientinnen.

Aus der Analyse der Beratungssituation im Rahmen der explorativen Interviews, der Interventionsmethodik und den obengenannten Punkten ist ersichtlich, dass es keine Methode der Wahl geben wird. Die Analyse der Beratung wird durch einen Methodenmix vorgenommen. Dieser wird sowohl einzelfallspezifische Instrumente wie auch Instrumente zur Erfassung der allgemeinen Beratungssituation umfassen.

Die Instrumente werden ausserdem der hohen Sensitivität des Themas und dem Patientinnenschutz gerecht und garantieren die möglichst grosse Offenheit der Untersuchungsteilnehmer.

\section{Weiteres Vorgehen}

In den kommenden Wochen erfolgt die Rekrutierung der teilnehmenden Ärztinnen und Ärzte durch die Frauenklinik in Zürich. Im Frühjahr 2004 wird die erste Analyse des Beratungsprozesses bei diesen Teilnehmern stattfinden. Die Einführung des Leitfadens wird im Rahmen einer Schulung im Mai 2004 in Zürich erfolgen. Im Anschluss daran wird die zweite Analyse bei den gleichen Teilnehmenden durchgeführt. Mitte Jahr wird der Prozess in Basel gestartet. Im nächsten Jahr erfolgt die Dokumentation der Studie. 


\section{Bedeutung der Untersuchung}

\section{Wissenschaftliche Bedeutung}

- Erforschung psychologischer Aspekte im Entscheidprozess in einer aus psychologischer Sicht herausfordernden Situation: Zeitdruck, Unsicherheit und über eigenes Leben hinausgehender Entscheidraum.

- Einzigartiger Beitrag zum Thema der psychologischen Aspekte in der Beratung im pränatalen Screening und in der Diagnostik über verschiedene Testangebote hinweg.

- Psychologischer Beitrag zum sehr aktuellen Thema Genetik und Umgang mit dem Thema aus psychologischer Sicht.

- Evaluation durch eine unabhängige dritte Partei (Abteilung für Angewandte Psychologie der Universität Zürich), welche in der Entwicklung des Beratungsinstrumentes nicht involviert war, garantiert eine unabhängige Analyse.

\section{Soziale und ökonomische Bedeutung}

- Dem Aspekt der Beratung soll mehr Gewicht verliehen werden. Die Debatte um die Legalisierung des Schwangerschaftsabbruchs hat jüngst aufgezeigt, dass das Bedürfnis hierfür besteht.

- Effizienter und strukturierter Ablauf in den Beratungsgesprächen.

- Sicherheitsaspekt für die Ärztinnen und Ärzte dank eines strukturierten Vorgehens (vgl. Haftpflichtfälle).

- Erstellung und Evaluation eines Schulungsmoduls für Ärztinnen und Ärzte, welches auf verschiedene Fachbereiche angewendet werden kann.
- Das Projekt soll zur Wertfreiheit in der Entscheidung verhelfen.

- Psychologischer Beitrag zum Thema Integration und Ausgrenzung in der Genetik.

- Das Know-how aus der Evaluation des Leitfadens fliesst in die geplanten Evaluationen der beiden andern Instrumente, der Broschüre für die Patientinnen und ihre Partner sowie des Films, ein.

\section{Literatur}

- DeLozier-Blanchet C, Wisser J. Prenatal diagnosis in Switzerland. Eur J Human Genetics 1997; 5:77-83

- Hildt E. Autonomy and freedom of choice in prenatal genetic diagnosis. Med Health Care Philosophy 2002;5:65-71.

- Marteau TM, Dormandy E. Facilitating informed choice in prenatal testing: how well are we doing? Am J Med Gen 2001;106:185-90.

- $\quad$ Rogers CR. Die nicht-direktive Beratung = counselling and psychotherapy. Frankfurt am Main: Fischer Taschenbuch; 1994.

- Schönholzer SM, Götzmann L. Psychosoziale Aspekte der Ultraschall-Untersuchung in der Schwangerschaft. Bern: Zentrum für Technologiefolgenabschätzung; 2001.

- Strauss RP. Beyond easy answers: prenatal diagnosis and counselling during pregnancy. Part of a panel discussion entitled "Beyond Easy Answers: Prenatal Diagnosis and Counselling During Pregnancy" at the 2000 Annual meeting of The American Cleft Parate-Craniofacial Association.

- Wolff G. Die Bedeutung von Beratung im Rahmen der pränatalen Diagnostik. Schweiz Med Wochenschr 1997; 127:60-8. 